\title{
Coupling of Polarization and Spatial Degrees of Freedom of Highly Divergent Emission in Broad-Area Square Vertical-Cavity Surface-Emitting Lasers
}

\author{
I. V. Babushkin, ${ }^{1}$ M. Schulz-Ruhtenberg, ${ }^{2}$ N. A. Loiko, ${ }^{3}$ K. F. Huang, ${ }^{4}$ and T. Ackemann ${ }^{5, *}$ \\ ${ }^{1}$ Max Born Institute for Nonlinear Optics and Short Pulse Spectroscopy, Max-Born-Str. 2a, 12489 Berlin, Germany \\ ${ }^{2}$ Institute for Applied Physics, University of Münster, Corrensstr. 2-4, 48149 Münster, Germany \\ ${ }^{3}$ Institute of Physics, NASB, 68 Nezalezhnasti Ave., 220072 Minsk, Belarus \\ ${ }^{4}$ Department of Electrophysics, National Chiao Tung University, Hsinchu, Taiwan \\ ${ }^{5}$ SUPA and Department of Physics, University of Strathclyde, Glasgow G4 ONG, Scotland
}

(Received 10 September 2007; published 29 May 2008)

\begin{abstract}
The polarization of highly divergent modes of broad-area square vertical-cavity surface-emitting lasers is shown to be only marginally affected by material anisotropies but determined by an interplay of the polarization properties of the Bragg cavity mirrors and of the transverse boundary conditions. This leads to a locking of the polarization direction to the boundaries and its indeterminacy for wave vectors oriented along the diagonal. We point out a non-Poissonian character of nearest-neighbor frequency spacing distribution and the impossibility of single-wave number solutions.
\end{abstract}

DOI: 10.1103/PhysRevLett.100.213901

Vertical-cavity surface-emitting lasers (VCSELs) are a special kind of semiconductor lasers which combine a high potential for applications with intricate dynamical behavior of fundamental interest in physics. The coherence of broad-area VCSELs is limited by the appearance of high order transverse modes $[1,2]$ being a manifestation of selforganization in nonequilibrium systems [3]. Particularly interesting is that these structures open also a simple access for studying the relation between wave and ray optics, quantum and classically trajectories, respectively, in billiard problems [4,5], where the reflective boundaries are created by refractive index steps determining the size of the active aperture. A further important aspect of selforganization in VCSELs stems from the fact that the ideal VCSEL is almost polarization isotropic and thus has an additional continuous phase variable leading to enhanced complexity. However, in small-area devices the polarization is closely related to the material anisotropies [6-9].

First observations in broad-area devices indicated that the emission consists of a few spots in far field with welldefined transverse wave vectors $\mathbf{k}_{\perp}$ with the polarization direction being orthogonal to $\mathbf{k}_{\perp}$ [1]. We will refer to this as the " $90^{\circ}$ rule" in the following. This tendency is due to an anisotropy appearing for off-axis waves due to a difference in reflection of $s$ and $p$ waves (which are polarized either perpendicular or parallel to $\mathbf{k}_{\perp}$ ) of the distributed Bragg reflectors (DBR) closing the cavity [10]. Later on, more complicated polarization configurations such as vortex scars were observed [11], but the physical reasons leading to these structures remained unclear.

In this work we shed light, for the first time up to our knowledge, on the mechanisms leading to complex polarization behavior of strongly off-axis emission in square VCSELs. We show that the polarization direction actually locks along the closest boundary. The structures having wave vectors along the diagonal $\left(k_{x} \approx k_{y}\right)$ become essen-
PACS numbers: 42.65.Sf, 05.45.Mt, 42.25.Ja, 42.60.Jf tially polarization degenerate. We interpret these intriguing observations by taking into account that reflections of waves at the side aperture and DBRs couple the polarization orientation of wave vectors already in linear order. Therefore, the $90^{\circ}$ rule reported in [1] is valid only in a tiny range of transverse wave vectors parallel to one of the boundaries. Our argument shows also that single-wave number solutions are not possible, which provides justification for the phenomenological construction of superposition states to explain the scared structures in [4]. The polarization degeneracy of the diagonal modes explains the possibility of locking between states with different polarization described in [11].

The linear framework of our considerations allows an interpretation of the threshold-states as arising from a wave billiard problem. It is known that the distribution of the frequency spacing between nearest-neighbor modes (i.e., the statistics of the mode spacing parameter $\delta \omega_{j}=$ $\omega_{j+1}-\omega_{j}$ for the modal eigenfrequencies $\omega_{j}$ ) is a good indicator of the level of integrability of the system. The eigenfrequencies of electromagnetic cavities used in microwave billiards [12] and microcavity lasers [13] with perfectly rectangular (or circular) boundaries follow a Poissonian distribution $p(\delta \omega) \sim e^{-\delta \omega}$, common for integrable systems. We show however that this is not true for square VCSELs, which makes the "vectorial VCSEL billiard" different from other types of optical cavities.

The devices under study are oxide-confined top-emitting VCSELs emitting at around $780 \mathrm{~nm}$. Their square apertures have a cross-section of $a=40 \mu \mathrm{m}$. The cavity, consisting of three $8 \mathrm{~nm}$ thick $\mathrm{Al}_{0.1} \mathrm{Ga}_{0.9}$ As quantum wells and $\mathrm{Al}_{0.4} \mathrm{Ga}_{0.6} \mathrm{As}$ spacer layers, is sandwiched by two highly reflective DBRs (top mirror: 31 layers, bottom mirror: 47 layers). The laser is mounted onto a heat sink with a feedback circuit and put into an air-tight box to allow temperature control of the device from about $40^{\circ}$ to $-35^{\circ} \mathrm{C}$. It is 
driven by a low noise dc current source. The laser beam is collimated by a microscope objective with a numerical aperture of 0.8. The near-field (image of the laser aperture) and the far-field (spatial Fourier spectrum) intensity distributions of the laser emission are detected by a cooled high-resolution charge-coupled device (CCD) camera. In order to allow control of the detected polarization component a half-wave plate and a linear polarizer are set into the beam path.

The setup allows measurement of spatial resolved Stokes parameters for the near-field and the far-field simultaneously. First checks, using a quarter-wave plate in addition, showed that the ellipticity is very small, being typically of the order of 0.02 . Hence, we will assume linear polarization in the following.

The main mechanism determining the length scale of spatial structures for broad-area devices is provided by the alignment of the cavity resonance and the gain maximum $[1,2]$. If the cavity resonance is redshifted versus the gain maximum (positive detuning $\delta>0$ ) tilted waves are favored to on-axis emission, which leads to pattern formation. In the experiment, $\delta$ can be changed by controlling the temperature of the laser [2,6]. Figure 1 shows experimental near-field and far-field images for three temperatures (detunings). The shown images are typical for almost all devices we studied.

For high temperature $(\delta \leq 0)$, emission occurs on-axis [Fig. 1(a)]. This corresponds to the situation in small-area VCSELs, where material anisotropies play the most important role in polarization selection at threshold. Thus, the polarization at $\mathbf{k}_{\perp} \approx 0$ is a good indicator for the intracavity anisotropy.

With increasing $\delta$, off-axis wave vectors $\mathbf{k}_{\perp}$ appear, which align parallel to one of the transverse oxide boundaries in tendency [see Fig. 1(b)]. At threshold, the resulting near-field structures are stripes, slowly modulated in the transverse direction. The far-field structure is dominated by four strong Fourier modes. At the same time, the polarization direction is approximately orthogonal to the dominant Cartesian component of the wave vector, i.e., seems to follow the $90^{\circ}$ rule. For increasing temperature the angle $\varphi$ of the wave vectors with the $x$ axis increases (Fig. 2) whereas the polarization vector remains close to the axis (except for $\mathbf{k}_{\perp}$ near the diagonal discussed below) as it is evidenced by the inset in Fig. 2, in clear violation of the $90^{\circ}$ rule. Deviations can be seen already in Fig. 1(b), where the polarization vector in the upper left (and the lower right) peak is at a larger angle than expected from the $90^{\circ}$ rule whereas the upper right one (and the lower left) has a smaller angle. Though the rotation of the polarization direction of the upper left peak could be explained by a dragging of the polarization vector towards the material anisotropy axis [denoted by the arrow in Fig. 1(a)], this is not the case for the upper right peak, because its polarization angle is smaller than the anisotropy angle. We will argue below that indeed the only significant influence of the anisotropy is to decide between the ideally symmetric (a)

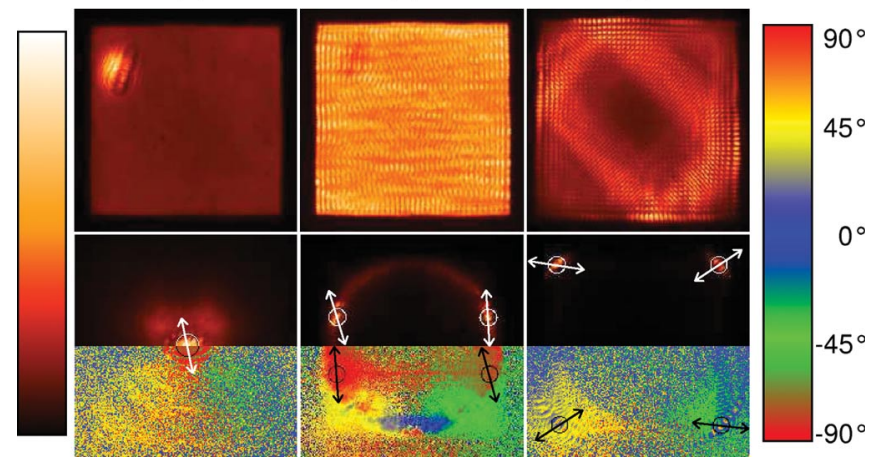

FIG. 1 (color). Illustration of the patterns appearing at different temperatures. (a) $\left(42^{\circ} \mathrm{C}, 12 \mathrm{~mA}\right.$, threshold current $I_{\text {th }}=$ $11.1 \mathrm{~mA}$ ): On-axis emission with polarization governed by the material anisotropies. (b) $\left(0{ }^{\circ} \mathrm{C}, 16 \mathrm{~mA} I_{\text {th }}=14.3 \mathrm{~mA}\right)$ : Wavy stripe patterns, consisting of four main Fourier modes near the axes. (c) $\left(-36^{\circ} \mathrm{C}, 22 \mathrm{~mA}, I_{\mathrm{th}}=19.5 \mathrm{~mA}\right)$ : Diamond-shaped patterns with the Fourier modes along the diagonal. Upper row: near-field intensity distribution, lower row: far-field; upper part: intensity distribution, lower part: polarization angle (the behavior is inversion symmetric to the origin). The average polarization of the intense peaks is indicated by the arrows, the area of averaging by the circles. The intensity is color coded in a temperaturelike scale with white denoting high intensities (left bar). Images of the polarization angle are color coded as shown by the right bar. All angles are given in the counterclockwise sense from the horizontal reference axis. Device size $40 \mu \mathrm{m}$, displayed range in far field $\left[-4.6,4.6 \mu \mathrm{m}^{-1}\right]$ in both directions.

configurations of the threshold modes having dominantly polarization along $\pm 90^{\circ}$ (and hence wave vectors along $x$ ) or along $0^{\circ}$ (and hence wave vectors along $y$ ). We note that the latter modes disfavored by the anisotropy often appear if the current is increased by a few $\mathrm{mA}$. There are visible in Fig. 2 as the data points with angles of $\mathbf{k}_{\perp}$ greater than $50^{\circ}$ (see the auxiliary material [14]).

For lower temperature (higher transverse wave number), the character of the structures in the near-field changes to a rather localized scar ("diamond", [4]), with the strongest Fourier components at the diagonal. The data presented in Fig. 2 allow an interpretation of this change of wave vector configuration: Obviously, for lower temperature and thus larger wave number the modes reach the cutoff of the waveguide (red line in Fig. 2) at some point, if they stay to be parallel to the boundaries. However, by moving to the diagonal, they can avoid cutoff.

The polarization structure of these diamonds is rather complex: We find components nearly parallel to the diagonal as well as ones parallel to the boundaries [Figs. 1(c) and 2]. There is even some gradient of polarization within the wings of a single peak (see the auxiliary material [14]). In other devices, all four peaks might have all main polarization components along the diagonal or all along the boundaries without any apparent regularity (see a few other examples in [14]). This lack of an apparent rule for polariza- 


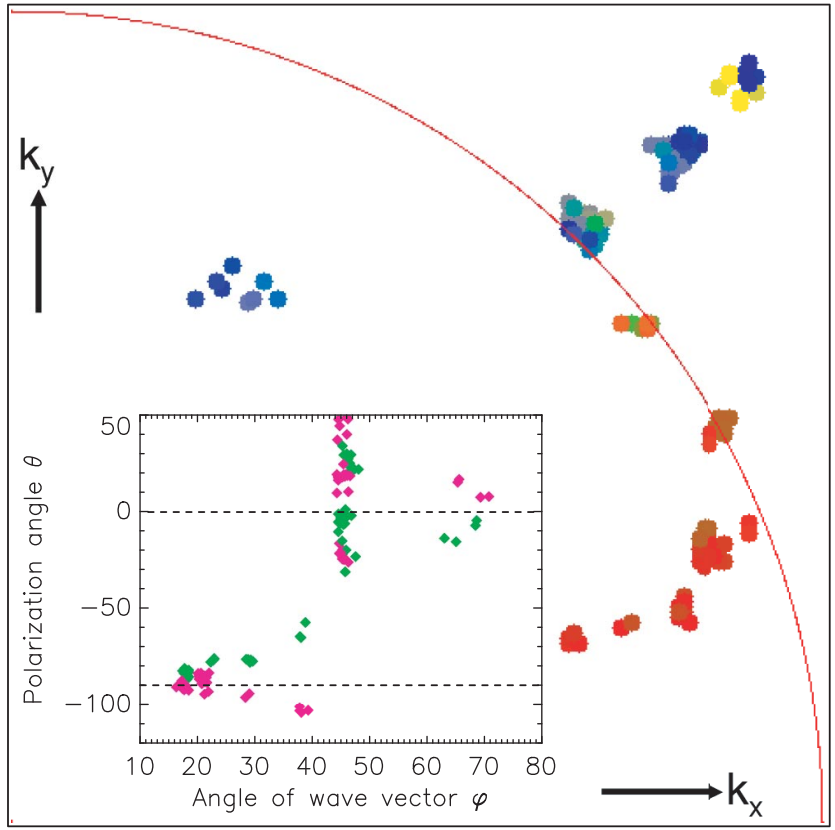

FIG. 2 (color). Mode map showing the polarization direction $\theta$ (color coded as in Fig. 1) for different wave vectors observed in dependence on temperature. The displayed range in the far field is $\left[0,3.6 \mu \mathrm{m}^{-1}\right]$ in both directions. The red line indicates the 1D cutoff of the oxide waveguide. The inset shows the dependence of $\theta$ on the absolute values of the angle $\varphi$ between the wave vector and the $x$ axis [magenta (green): peaks from first (second) quadrant]. The levels of $0^{\circ}$ and $90^{\circ}$ are marked by dashed lines.

tion selection indicates that the polarization is highly degenerate in the vicinity of the diagonal.

In order to analyze the behavior described above we use a model for a broad-area VCSEL which accounts for its cavity structure including DBRs [10]. As shown in [10,15], the cylindrical symmetry of a VCSEL is broken for off-axis waves. Hence, many features of pattern selection are already obtained by a linear stability analysis. Below, we present only the main milestones of the analysis, the technical details can be found in the auxiliary material [14].

For the spatially homogeneous device with infinite aperture, the eigenmodes are plane transverse waves $\mathbf{E}(x, y, t)=\mathbf{e}(\mathbf{t}) \exp \left\{i\left(k_{x} x+k_{y} y\right)\right\}, \quad$ where $\quad \mathbf{E}(x, y, t) \equiv$ $\left\{E_{x}, E_{y}\right\}$ is the slowly varying complex envelope of the field inside the cavity. Linearization of the laser equations at lasing threshold leads to an eigenvalue problem

$\beta\left(\mathbf{k}_{\perp}\right) \mathbf{e}=\lambda \mathbf{e}$ with $\beta=\Gamma+i a k_{\perp}^{2}+i b s\left(\mathbf{k}_{\perp}\right)-\kappa \Upsilon\left(\mathbf{k}_{\perp}\right)$.

$\beta\left(\mathbf{k}_{\perp}\right)$ is a $2 \times 2$ matrix (with $\mathbf{k}_{\perp}$-dependent coefficients) describing the polarization states. It includes the material anisotropy $\Gamma$, the diffraction both in the cavity $i a k_{\perp}^{2}$ and in the DBRs $i b s\left(\mathbf{k}_{\perp}\right)$ ( $a$ and $b$ are some coefficients, $s$ is the phase shift due to reflection from the DBRs), as well as the losses and the gain $\kappa Y\left(\mathbf{k}_{\perp}\right)$ ( $\kappa$ is the field decay rate). $\Gamma$ includes amplitude $\left(\gamma_{a}\right)$ and phase $\left(\gamma_{p}\right)$ anisotropies and is described by a matrix, diagonal in their principal axes coordinate system $\Gamma=\operatorname{diag}\left(\gamma_{a}+i \gamma_{p},-\gamma_{a}-i \gamma_{p}\right)$. The other source of anisotropy is provided by $Y\left(\mathbf{k}_{\perp}\right)$, which includes the matrix of reflection $R$ from the DBRs. $R$ is diagonal in the basis of $s$ - and $p$-waves: $R=\operatorname{diag}\left(r_{s}, r_{p}\right)$. Because for $k_{\perp}>0\left|r_{s}\right|>\left|r_{p}\right|$, an $s$ wave is preferred at threshold, which is the origin of the $90^{\circ}$ rule [10].

To describe the finite device, we rewrite $\beta$ in terms of the modes of the waveguide formed by the oxide refractive index steps at the side boundaries. For a device with an aperture length of $a$, these are $\mathbf{E}_{n m}^{(x)}=f_{n}(\pi x / a) \times$ $f_{m}(\pi y / a) \mathbf{n}_{x}, \mathbf{E}_{n m}^{(y)}=f_{n}(\pi x / a) f_{m}(\pi y / a) \mathbf{n}_{y}$, where $\mathbf{n}_{x}, \mathbf{n}_{y}$ are unit vectors in the corresponding directions, $f_{n}(p)=$ $\cos (n p)$ if $n$ is even and $\sin (n p)$ if $n$ is odd. In far field they are represented by four wave vectors $\left\{\left(k_{x}, k_{y}\right)\right.$, $\left.\left(k_{x},-k_{y}\right),\left(-k_{x}, k_{y}\right),\left(-k_{x},-k_{y}\right)\right\}$ with equal field amplitudes and polarization directions.

It is important to realize that these modes are not eigenmodes of the reflection of the DBR because the incident waveguide mode cannot represent a pure $s$ or $p$ wave simultaneously for all the constituent wave vectors. For example, for the case of $\mathbf{E}_{2 n, 2 m}^{(x)}=\mathbf{n}_{x} \cos (2 \pi n x / a) \times$ $\cos (2 \pi m y / a)$ being incident onto the DBR, elementary calculations show that the reflected wave has a component proportional to $\mathbf{n}_{y}\left(r_{p}-r_{s}\right) \sin (2 \pi n x / a) \sin (2 \pi m y / a)$, which is not an eigenmode of the waveguide. Mathematically, it can be represented as a sum of waveguide modes with different $\left|\mathbf{k}_{\perp}\right|$, which physically means that such a component is rescattered into the other waveguide modes. The rescattered modes can be seen in the experimental pictures [especially in the lower part of Fig. 1(c), Fig. 3 of the auxiliary material [14], and in Fig. 4 of [4] and Fig. 2 of [1]]. This rescattering is essentially a polarization effect because it disappears for $r_{p}=r_{s}$. The scattering process provides a seed, invalidating true single-wave number solutions expected in simpler laser models $[10,16]$ and thus can be at the origin of the more complex wave vector configurations found experimentally and described phenomenologically before as superposition states of off-axis waves centered around a certain wave number [4].

By rewriting $\beta$ in the terms of waveguide modes we obtain an operator $\beta_{s}$, acting on the whole mode set since all the modes are coupled by the rescattering. However, for a first analysis of the polarization behavior we neglect the rescattered modes in $\beta_{s}$ and thus obtain a set of $2 \times 2$ matrices $\beta_{p}\left(k_{x}, k_{y}\right)$ (acting on the pairs of modes $\mathbf{E}_{n m}^{(x)}, \mathbf{E}_{n m}^{(y)}$ with the corresponding $k_{x}, k_{y}$ ). The eigenvector of $\beta_{p}$ with the largest real part is shown in Fig. 3 (the second one is always perpendicularly polarized).

The polarization state at $\mathbf{k}_{\perp}=0$ is determined by the intracavity anisotropy, which was adjusted to match the experimental observation. For larger $k_{\perp}$, the polarization locks to the nearest axes due to the influence of the boundaries. This locking induces a rather abrupt transition close to the diagonal. In addition, at the diagonal the two branches of eigenvalues intersect [see the inset in Fig. 3(b)] 

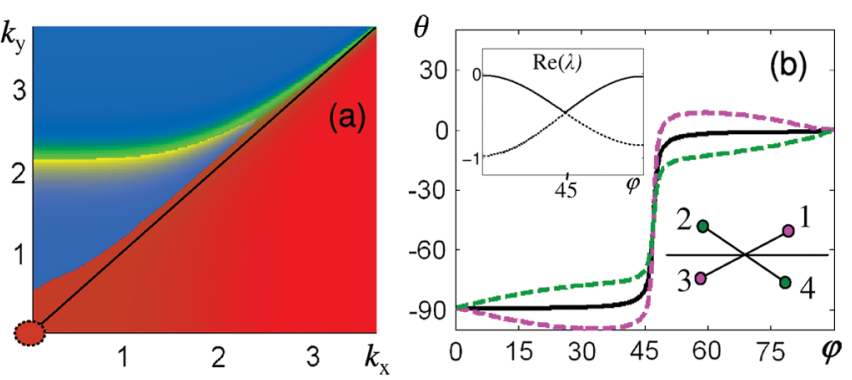

FIG. 3 (color). Theoretical analysis of polarization selection neglecting the rescattered modes. (a) - the polarization direction $\theta$ of the eigenmode of $\beta_{p}$ with maximal $\operatorname{Re}(\lambda)$ versus $\mathbf{k}_{\perp}$ inside the cavity for the first quadrant [spot 1 in the right inset in (b)]. The color coding is shown in Fig. 1. The diagonal $\left(k_{x}=k_{y}\right)$ is highlighted by a thin black line. (b) Cross section of (a) (solid black line) along the circle with $\left|k_{\perp}\right|=3.1 \mu \mathrm{m}^{-1}$. The polarization directions outside of the cavity for the first and second quadrant (spots 1 and 2 in the right inset illustrating the wave vector configuration) are shown by magenta and green dashed lines. The left inset shows $\operatorname{Re}(\lambda)$ versus the wave vector angle for two eigenmodes (with orthogonal polarization). Polarization anisotropies taken for calculations are $\gamma_{p}=60 \mathrm{~ns}^{-1}, \gamma_{a}=$ $-0.1 \mathrm{~ns}^{-1}$ with the principal axis being at $-78^{\circ}$. The circle at the origin in (a) emphasizes the corresponding color.

and the polarization modes are degenerate. This explains the possibility of frequency locking between diamond states of orthogonal polarization reported in [11]. The degeneracy is evidenced experimentally by the continuum of polarization angles between about $-40^{\circ}$ and $45^{\circ}$. The wave vectors with $\theta \gtrsim 10^{\circ}$ present in Fig. 2 but not in Fig. 3(b) are interpreted to stem from the nearly degenerate orthogonally polarized branch of eigenvectors.

The polarization state of the extra-cavity field is different from the intracavity one due to the final transition through the DBR. In transmission, $p$-wave components are favored. Hence the polarization state of the transmitted light is rotated towards the wave vector (see the dashed lines in Fig. 3(b)]. The polarization of wave vectors in neighboring quadrants is therefore slightly different, which is also obvious in the experiment [Fig. 1(a)]. The inset in Fig. 2 also shows nicely the splitting present for the dashed lines in Fig. 3(b) and their remerging close to the diagonal $\left(\varphi \approx 45^{\circ}\right)$.

Finally, we can interpret the linear operator $\beta_{s}$ describing the onset of pattern formation also as an operator of a quantum billiard problem. In our case the operator $\beta_{s}$ contains not only the kinetic energy term $\sim k_{\perp}^{2}$, but the complex linear coupling terms due to the vectorial degrees of freedom. First calculations indicate that the solution of the eigenproblem for $\beta_{s}$-now including the rescattered modes - shows a clear deviation of the nearest-neighbor eigenfrequency spacing distribution from a Poissonian one despite the fact that the device boundaries are assumed to be perfectly square. Such a distribution is similar to one present in a certain class of pseudointegrable billiards [17], i.e., ones which are in between fully integrable and fully chaotic ones. This provides a promising starting point for further investigations.

In conclusion, we have shown that the main mechanism for polarization direction selection for strongly off-axis emission in square VCSELs is related to the interaction of the reflection from the DBR and from the side boundaries, leading to rescattering into neighboring transverse modes with different wave number and providing dynamical justification for the construction of superposition states in [4]. This leads to a locking behavior of the polarization axis at the boundaries and correspondingly to an indeterminacy of polarization state for modes at the diagonal.

We acknowledge financial support from the Deutsche Forschungsgemeinschaft, the Deutsche Akademische Austauschdienst and the Taiwanese Research Council (grant No. NSC-96-2112-M-009-027-MY3). T. A. is grateful for useful discussions with J. V. Moloney.

*Corresponding author. thorsten.ackemann@strath.ac.uk

[1] S. P. Hegarty, G. Huyet, J.G. McInerney, and K. D. Choquette, Phys. Rev. Lett. 82, 1434 (1999).

[2] M. Schulz-Ruhtenberg et al., Appl. Phys. B 81, 945 (2005).

[3] M. C. Cross and P. C. Hohenberg, Rev. Mod. Phys. 65, 851 (1993); F. T. Arecchi, S. Boccaletti, and P. L. Ramazza, Phys. Rep. 318, 1 (1999).

[4] K.F. Huang, Y.F. Chen, H. C. Lai, and Y. P. Lan, Phys. Rev. Lett. 89, 224102 (2002); Y. F. Chen, K. F. Huang, and Y. P. Lan, Phys. Rev. E 66, 046215 (2002).

[5] T. Gensty et al., Phys. Rev. Lett. 94, 233901 (2005).

[6] K.D. Choquette et al., IEEE J. Sel. Top. Quantum Electron. 1, 661 (1995).

[7] M. SanMiguel, Q. Feng, and J. V. Moloney, Phys. Rev. A 52, 1728 (1995).

[8] M. P. vanExter, A. Al-Remawi, and J. P. Woerdman, Phys. Rev. Lett. 80, 4875 (1998).

[9] T. Ackemann and M. Sondermann, Appl. Phys. Lett. 78, 3574 (2001).

[10] N. A. Loiko and I. V. Babushkin, J. Opt. B 3, S234 (2001).

[11] Y.F. Chen, K. F. Huang, H.C. Lai, and Y. P. Lan, Phys. Rev. Lett. 90, 053904 (2003).

[12] H.-J. Stoeckmann, Quantum Chaos: An Introduction (Cambridge University Press, Cambridge, 1999).

[13] J. U. Nöckel and D. Stone, Nature (London) 385, 45 (1997); C. Gmachl et al., Science 280, 1556 (1998).

[14] See EPAPS Document No. E-PRLTAO-100-002822 for auxiliary material. For more information on EPAPS, see http://www.aip.org/pubservs/epaps.html.

[15] I. V. Babushkin, N. A. Loiko, and T. Ackemann, Phys. Rev. E 69, 066205 (2004).

[16] P. K. Jakobsen, J. V. Moloney, A. C. Newell, and R. Indik, Phys. Rev. A 45, 8129 (1992).

[17] E. Bogomolny and C. Schmit, Phys. Rev. Lett. 93, 254102 (2004); E. Bogomolny et al., Phys. Rev. Lett. 97, 254102 (2006). 